\title{
ESTUDO DA INTERAÇÃO DE METAIS (Pb E Cd) COM SUBSTÂNCIAS HÚMICAS DE TURFA
}

\author{
STUDY OF THE INTERACTION OF METALS (PB AND CD) WITH HUMIC \\ SUBSTANCES OF PEAT
}

Vinícius Marques Gomes ${ }^{1}$; Camila Garcia Matheus Santiago ${ }^{2}$; Cristiane Hernandes Lopes ${ }^{3}$; Daniel Ângelo Macena ${ }^{4}$; 1 Universidade do Oeste Paulista - Unoeste,Faculdade de Ciências, Letras e Educação
de Presidente Prudente - FACLEPP.
$\begin{aligned} & \text { E-mail: viniciusmarques@unoeste.br1 ; } \quad \text { milagaarcia2@gmail.com2; } \\ & \text { crikahl14@hotmail.com3; daniel@unoeste.br4 }\end{aligned}$

RESUMO - Dentre os poluentes indicadores de contaminação ambiental, os metais Cádmio ( $\mathrm{Cd}$ ) e Chumbo $(\mathrm{Pb})$ são preocupantes. A interação desses metais com as substâncias húmicas (principais constituintes da matéria orgânica natural) resulta em reações de complexação e/ou redução. O propósito deste trabalho foi estudar a interação existente entre substâncias húmicas de turfa e diferentes metais $(\mathrm{Cd})$ e $(\mathrm{Pb})$ potencialmente tóxicos. Os resultados desse trabalho mostraram que a matéria orgânica está exercendo uma importante função, complexando os íons metálicos e agindo como uma barreira de proteção natural. Conclui-se que os riscos do descarte indiscriminado de resíduos contendo metais potencialmente tóxicos ao solo, estão associados principalmente ao transporte e destinação desses poluentes no ambiente e a natureza estrutural das substâncias húmicas em estudo favoreceu a complexação dos íons metálicos introduzidos antropicamente no ambiente.

Palavras-chave: complexação; matéria orgânica; metais tóxicos.

Recebido em: 20/12/2016

Revisado em: 20/04/2017

Aprovado em: 10/05/2017
ABSTRACT - Among the indicators of environmental contamination, metals Cadmium $(\mathrm{Cd})$, lead $(\mathrm{Pb})$ are worrying. The interaction of these metals with humic substances (main constituent of natural organic matter) results in complexation reactions or reduction. The purpose of this work was to study the interaction between humic substances of peat and different metals $(\mathrm{Cd})$ and $(\mathrm{Pb})$ potentially toxic. The results of this work showed that the organic matter played an important role, complexing metal ions and acting as a natural protective barrier. It follows that the risks of indiscriminate disposal of waste-containing soil potentially toxic metals are mainly associated with the transportation and disposal of these pollutants into the environment and structural nature of humic substances under examination favors the complexation of metal ions anthropically introduced into the environment.

Keywords: complexation; organic matter; potentially toxic metals. 


\section{INTRODUÇÃO}

Nos últimos anos, há uma maior preocupação com a qualidade do ambiente. Pôde-se notar que o problema mais sério que afeta o ambiente é a poluição química, tanto orgânica, quanto inorgânica, gerada a partir de despejos industriais e residenciais, portanto, a poluição química é classificada como um dos contaminantes do solo, por lixiviação, tornando a água imprópria para o consumo humano (DERISIO, 2016).

Dentre os contaminantes químicos está presente o Cádmio (Cd) e o Chumbo $(\mathrm{Pb})$, que são metais potencialmente tóxicos considerados metais pesados e/ou elementos traços. A preocupação quanto ao ambiente contaminado ou exposição por esses metais é que podem causar sérios danos aos seres vivos por serem bioacumulativos, além de tais danos serem graves e por outro lado, muitas vezes irreversíveis (NATUME, 2011).

O Cádmio, afeta os humanos através de alimentos, água contaminada e por inalação, sendo que os fumantes são os mais afetados, já que o cigarro pode conter certa quantidade desse metal. Se ingerido, pode provocar náuseas, vômitos, diarreias e dores abdominais; por inalação em curto prazo é capaz de ocasionar à traqueobronquite, pneumonite química e agravar ao edema pulmonar, devido à destruição das células epiteliais do pulmão, além de ser um elemento carcinogênico para o ser humano, com base em evidência de tumores pulmonares em trabalhadores e animais expostos por via inalatória (CETESB, 2012a).

De acordo com a Companhia Ambiental do Estado de São Paulo - CETESB (2012b), o chumbo também pode ser absorvido pelo organismo por inalação e ingestão, afetando quase todos os órgãos, sendo o sistema nervoso central o alvo principal, tanto em crianças quanto em adultos. Os principais efeitos da contaminação por chumbo são: fraqueza, irritabilidade, astenia, náusea, dor abdominal com constipação e anemia, além de também ser considerado carcinogênico. Os compostos orgânicos de chumbo, como chumbo tetraetila e tetrametila, se comportam como gases no trato respiratório e são mais absorvidos que as partículas de chumbo inorgânico, sendo assim, absorvido através da pele. O chumbo pode ser encontrado também em cosméticos e no cigarro (MOREIRA; MOREIRA, 2004).

Além dos meios citados, esses metais podem ser encontrados em produtos agrícolas, contaminando assim o solo e a plantação, colocando em risco o bem estar da população por serem potencialmente tóxicas, carcinogênicas ou mutagênicas (OLIVEIRA et al., 2016).

Nos solos, está presente a matéria orgânica (M.O) em consequência da atividade dos seres vivos. É constituída pela 
mistura de microrganismos e resíduos de vegetais e animais os quais, devido à degradação química e biológica, encontra-se em vários estágios de decomposição. Desta forma, os tecidos das plantas superiores são fontes primárias não só de alimento para os diversos organismos, mas também de matéria orgânica, essencial à formação do solo (GOMES; SANTOS; OLIVEIRA, 2010).

As substâncias húmicas (SH) são os principais constituintes da matéria orgânica natural (MON), globalmente distribuída em ambientes terrestres e aquáticos. Estima-se que cerca de $50 \%$ do carbono orgânico dissolvido (COD) em águas superficiais e oceânicas consiste em matéria orgânica refratária do tipo SH (ROCHA; ROSA; CARDOSO, 2009).

As $\mathrm{SH}$ dividem-se em três principais frações: os ácidos húmicos definidos operacionalmente como a fração das SH solúvel em meio alcalino diluído e que precipita pela acidificação do extrato alcalino. Os ácidos fúlvicos que permanecem em solução quando o extrato alcalino é acidificado e a humina que é a fração não extraída por ácido ou álcali diluído (BOTERO; SANTOS; ROCHA, 2008).

Para a formação das SH existe todo um processo a partir da decomposição dos resíduos no solo, onde, inicia-se pelo processo principal que é a oxidação de substratos hidrolisados monoméricos (Figura $1)$.

Figura 1. Mecanismo das principais vias de formação das SH.

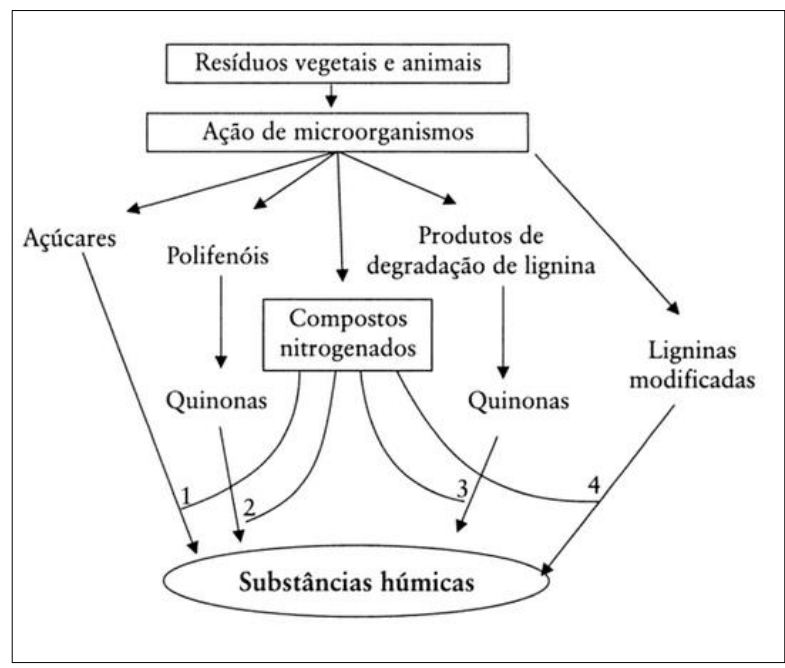

Fonte: (ROCHA; ROSA, 2003).

As SH são ambientalmente importantes principalmente pela sua coloração escura que possibilita a retenção de calor pelo solo, beneficiando a germinação de sementes e o desenvolvimento de raízes, influenciam a biodisponibilidade de metais do solo para plantas e/ou organismos da micro e macro fauna, possuem alta capacidade de retenção de água, influem na toxicidade de alguns metais, formando complexos com diferentes labilidades relativas (ROCHA; ROSA, 2003).

A interação com espécies metálicas no ambiente é uma importante propriedade das $\mathrm{SH}$, a qual resulta em reações de complexação e/ou redução. As reações de complexação de SH com metais têm sido investigadas por influenciar na 
biodisponibilidade dessas espécies (SANTOS, 2003)

Devido ao alto teor de oxigênio encontrado na estrutura das $\mathrm{SH}$, elas têm excepcional capacidade para complexação de metais (SANTOS, 2003).

Esta propriedade de interagir com íons metálicos para formar complexos de diferentes estabilidades e características estruturais, tem sido objetivo de estudos para pesquisadores (ROCHA; ROSA, 2003).

De acordo com Gomes, Santos e Oliveira (2010), a capacidade das SH de reter elementos é tão importante que pode influenciar diretamente no aquecimento global por ser uma forma de acúmulo de carbono, armazenando o $\mathrm{CO}_{2}$ e contribuindo para minimizar o aquecimento global. Além disso, a alta capacidade complexante das SH pode alterar a biodisponibilidade e os efeitos toxicológicos de metais em sistemas aquáticos. Recentes investigações concluíram que, para caracterizar a estabilidade da ligação entre espécies metálicas e SH, são necessários procedimentos analíticos adequados que forneçam importantes informações.

Devido à importância da capacidade de complexação das SH e íons metálicos apresentados, este trabalho teve por objetivo avaliar a interação entre as SH e metais Cd e $\mathrm{Pb}$., metais considerados potencialmente tóxicos e comparar outros estudos da literatura que obteve como finalidade mostrar a interação SH-metal por meio de outra técnica.

\section{MATERIAL E MÉTODOS}

\subsection{Aquisição e preparo da amostra}

Para o preparo da amostra utilizou-se turfa adquirida em loja de materiais de paisagismo, onde o mesmo foi pulverizado com almofariz e pistilo, e passado por uma peneira comum. Em seguida foi pesado $100 \mathrm{~g}$ de amostra de Turfa e transferida para um béquer de $500 \mathrm{~mL}$.

\subsection{Extração das Substâncias Húmicas (SH)}

Para extração das substâncias húmicas, utilizou-se da metodologia recomendada pela Sociedade Internacional e Substâncias Húmicas - IHSS, onde, em 100 g de Turfa pesada anteriormente, adicionou-se solução de Hidróxido de Sódio $(\mathrm{NaOH})$ 0,1 M na razão 1:10 (m/v) e a mistura passou por agitação mecânica durante 4 horas, com velocidade $550 \mathrm{rpm}$ à $23^{\circ} \mathrm{C}$ e depois centrifugada (ROSA et al., 1998; GOMES; SANTOS; OLIVEIRA, 2010).

Após 30 minutos de centrifugação foram utilizados apenas o sobrenadante ao qual corresponde as substâncias húmicas (Figura 2).

Após a centrifugação e separação do sobrenadante $(\mathrm{SH})$, fez-se o ajuste de $\mathrm{pH}$ 
utilizando solução de Ácido Clorídrico (HCl) 1 M, ajustando todos os pH's próximos de 7 .

Figura 2. Amostra após a etapa de centrifugação (sobrenadante $-\mathrm{SH}$ ).

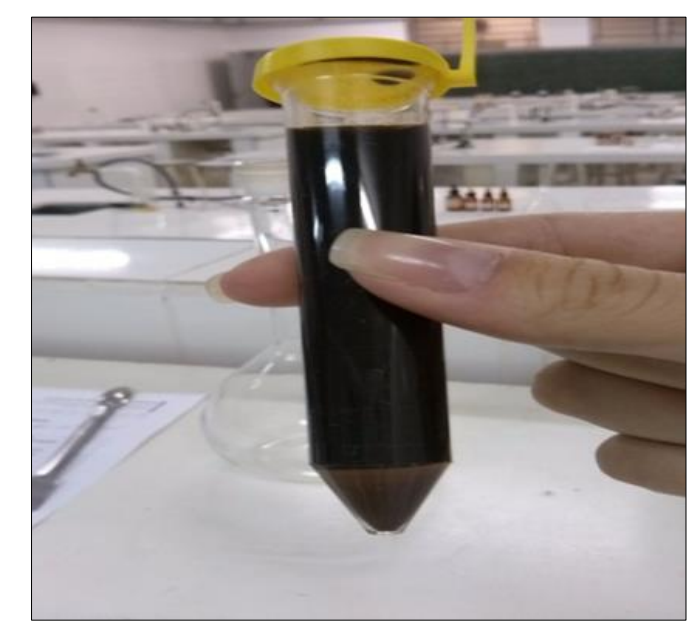

Fonte: Próprio autor.

2.3. Determinação da capacidade de complexação das SH

Para o estudo de complexação entre as espécies metálicas e substâncias húmicas, utilizou-se o seguinte procedimento analítico: pipetou-se a primeira alíquota $(5 \mathrm{~mL})$, a qual corresponde ao tempo zero, ou seja, antes da adição da solução padrão de metal. Com uma pipeta automática, foram feitas 9 adições de $1 \mathrm{~mL}$ da solução metálica de concentração $600 \mathrm{mg} \cdot \mathrm{L}^{-1}$, à $250 \mathrm{~mL}$ de solução de SH correspondente à $\left.100 \mathrm{mg} \cdot \mathrm{L}^{-1}\right)$. A cada adição, fez-se agitação da mistura por 10 minutos (tempo estimado seguindo protocolo utilizado por Gomes, Santos e Oliveira (2010) no estudo de complexação de SH e Crômio) para que fosse possível a formação do complexo SH-metal, Em seguida, retirava-se da mistura, uma alíquota de $5 \mathrm{~mL}$ a cada adição no sentido de transferir para trouxas de celofane mergulhada em volume conhecido de água. O celofane possui poros muito pequenos que permite a passagem do metal livre, mas não permite a passagem do complexo SH-metal.

No momento em que os sítios ativos das SH estão totalmente preenchidos com átomos metálicos, espera-se que o metal livre (não complexado) ultrapasse a barreira de celofane e possa ser determinado no volume conhecido de água por espectrometria de absorção atômica com atomização por chama.

A capacidade de complexação das SH é convencionalmente expressa em $\mathrm{mmol}$ de metal por grama de $\mathrm{SH}$ e caracteriza a máxima quantidade de metais, que podem ser complexados às $\mathrm{SH}$ em solução aquosa. Assim, trata-se de um importante parâmetro de qualidade ambiental, pois devido a esta capacidade das $\mathrm{SH}$, a disponibilidade de várias espécies para participar de reações no ambiente, incluindo metais, é minimizada.

\subsection{Determinação das espécies metálicas}

As curvas de calibração para a determinação das espécies metálicas foram realizadas a partir de diluições de soluções concentradas. Preparou-se uma curva de calibração para cada espécie metálica, com 
cinco (05) pontos de concentrações entre $0,10-5,00 \mathrm{mg} \cdot \mathrm{L}^{-1}$.

As concentrações das espécies metálicas foram determinadas por espectrometria de absorção atômica em chama (FAAS) da marca Perkin Elmer modelo AAnalyst 200, técnica implantada em laboratórios para análises químicas em larga escala (CADORE; MATOSO; SANTOS, 2008).

\section{RESULTADOS E DISCUSSÃO}

\subsection{Substâncias húmicas com Cádmio}

A Figura 3 apresenta a curva traçada para a determinação da capacidade complexante entre SH-Cd. Ela apresenta uma mudança de inclinação e a capacidade de complexação foi obtida pela intersecção das duas seções lineares da mesma (GOMES; SANTOS; OLIVEIRA, 2010).

Figura 3. Capacidade de complexação do Cádmio por SH de turfa.

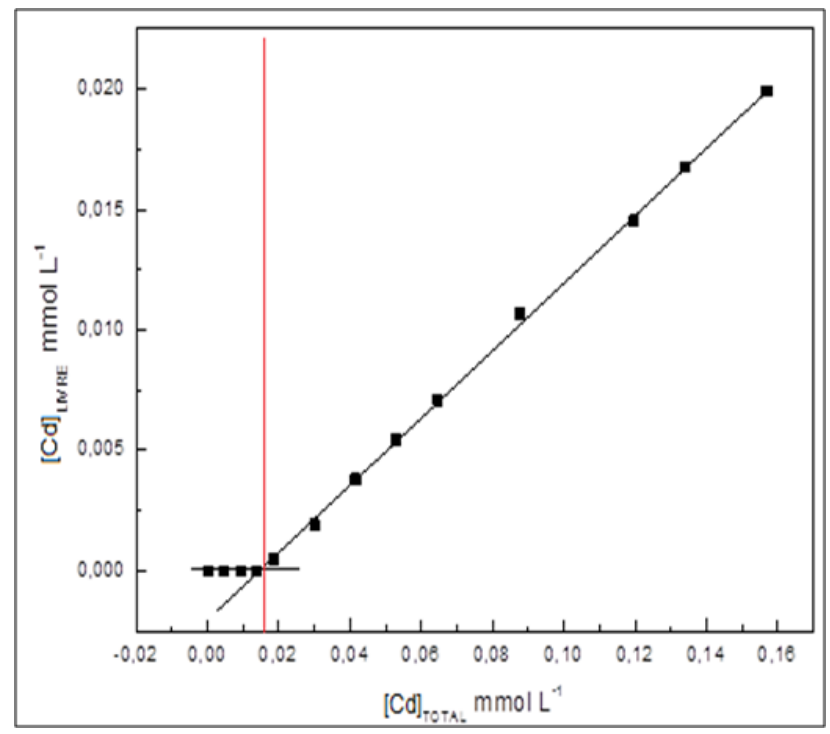

Fonte: Dados experimentais

A interação com espécies metálicas no ambiente é uma importante propriedade das $\mathrm{SH}$, a qual resulta em reações de complexação e/ou redução. As reações de complexação de SH com metais têm sido investigadas por influenciar na biodisponibilidade dessas espécies (SANTOS, 2003).

Nos três primeiros pontos da Figura 3, não houve detecção de Cádmio livre nos filtrados. Portanto, pode-se inferir que nessas concentrações o metal está sendo totalmente complexado pelas $\mathrm{SH}$.

A partir dessa fase, inicia-se a detecção de íons livres nos respectivos filtrados, indicando que a capacidade de formar espécies SH-Cd foi atingida e, consequentemente não há mais sítios livres disponíveis para complexar $\mathrm{Cd}$ nas $\mathrm{SH}$.

Considerando a intersecção dos dois segmentos de retas da Figura 3, determinouse que a capacidade de complexação da SH 
com o íon Cd, é de $0,624 \mathrm{mmol} \mathrm{Cd} \mathrm{g}^{-1}$ de SH. Resultado obtido após conversão da concentração de $\mathrm{Cd}$ passando de $\mathrm{mmol} \mathrm{L}^{-1}$ para mmol de massa específica por g de $\mathrm{SH}$.

Segundo Pearson (1963), as espécies químicas podem ser classificadas como ácidos e bases "duros" e "moles" e os conceitos de "dureza" e "maciez" ajudam a interpretar parte dos mecanismos envolvidos nas reações de complexação. A tendência de os ácidos "moles" ligarem-se às bases "moles" e a dos ácidos "duros" ligarem-se às bases "duras", explica a forma que os elementos são encontrados na crosta terrestre. Nesta classificação existem duas classes principais: os elementos litofílicos e os elementos calcofílicos. Os elementos litofílicos são cátions duros, entre eles o magnésio, alumínio, lítio, cálcio, ferro, manganês e crômio possuindo grande afinidade por bases duras, como $\mathrm{O}^{2-}$. Por outro lado os elementos calcofílicos (ácidos moles) são frequentemente encontrados em combinação com grupos aminos e cianos (bases moles) e incluem cobre, chumbo, mercúrio, zinco, cádmio, dentre outros.

Tabela 1. Capacidade complexante das SH de turfa comparadas a amostras da literatura.

\begin{tabular}{|c|c|c|c|c|}
\hline \multirow{2}{*}{ Amostras } & \multicolumn{4}{|c|}{$\begin{array}{l}\text { Espécies metálicas / Capacidade complexante } \\
\left.\text { (mmol metal } \mathrm{g}^{-1} \mathrm{SH}\right)\end{array}$} \\
\hline & $\mathrm{Cd}$ & $\mathrm{Mg}$ & $\mathrm{Ca}$ & $\mathrm{Cu}$ \\
\hline Este estudo & 0,62 & --- & --- & --- \\
\hline MENDONÇA, 2010 & --- & 0,69 & 0,61 & 0,51 \\
\hline ROMÃO et al., 2003 & --- & --- & --- & 0,38 \\
\hline
\end{tabular}

Comparando o resultado obtido com dados da literatura (Tabela 1) observa-se que a capacidade complexante obtida para $\mathrm{Cd}$ neste estudo, corrobora com resultados obtidos também para outros cátions duros (Ca e Mg), com substâncias húmicas de característica estrutural similar à amostra em estudo. Essa maior afinidade provavelmente esta relacionada com a grande quantidade de grupos oxigenados (bases "duras") presentes nas amostras de SH. A menor afinidade por ácidos moles (cobre, por exemplo) está associada provavelmente ao menor teor de 
grupos nitrogenados e ciano presente nessas $\mathrm{SH}$.

\subsection{Substâncias húmicas com Chumbo}

A Figura 4 apresenta a curva traçada para a determinação da capacidade complexante entre substâncias húmicas e Chumbo. Ela apresenta uma mudança de inclinação e a capacidade de complexação foi obtida pela intersecção das duas seções lineares da mesma (GOMES; SANTOS; OLIVEIRA, 2010).

Para a amostra SH-Chumbo, de acordo com a Figura 4, não houve detecção de chumbo livre nos filtrados dos dois primeiros pontos, ou seja, novamente nessas concentrações o metal está sendo totalmente complexado pelas $\mathrm{SH}$.

A partir dessa fase, inicia-se a detecção de íons livres nos respectivos filtrados, indicando que a capacidade de formar espécies SH-Chumbo foi atingida e, consequentemente não há mais sítios livres disponíveis para complexar chumbo nas $\mathrm{SH}$.

Considerando a intersecção dos dois segmentos de retas da Figura 4, determinou- se que a capacidade de complexação da SH com o íon chumbo é de 0,249 $\mathrm{mmol} \mathrm{Pbg}^{-1}$ de $\mathrm{SH}$.

Desta maneira, verifica-se que existe uma diferença significativa entre as capacidades de complexação do cadmio e do chumbo, ou seja, a amostra possui um número muito menor de sítios livres que acomodam os íons de chumbo (átomo maior).

Bezerra, Takiyama e Bezerra (2009) contribuíram com outro estudo sobre determinar a capacidade da matéria orgânica dissolvida em complexar os íons metálicos Cobre; Cádmio e Chumbo, em amostras de águas superficiais de rios que integram a Amazônia legal. Concluíram que a presença de metais na forma livre em corpos aquáticos é controlada pelo sítio preferencial de complexação e analiticamente os autores mostraram que o $\mathrm{Pb}$ é o íon metálico que numa ordem dos metais em estudo possui a maior capacidade de complexação. 
Figura 4. Capacidade de complexação do Chumbo por SH de turfa.

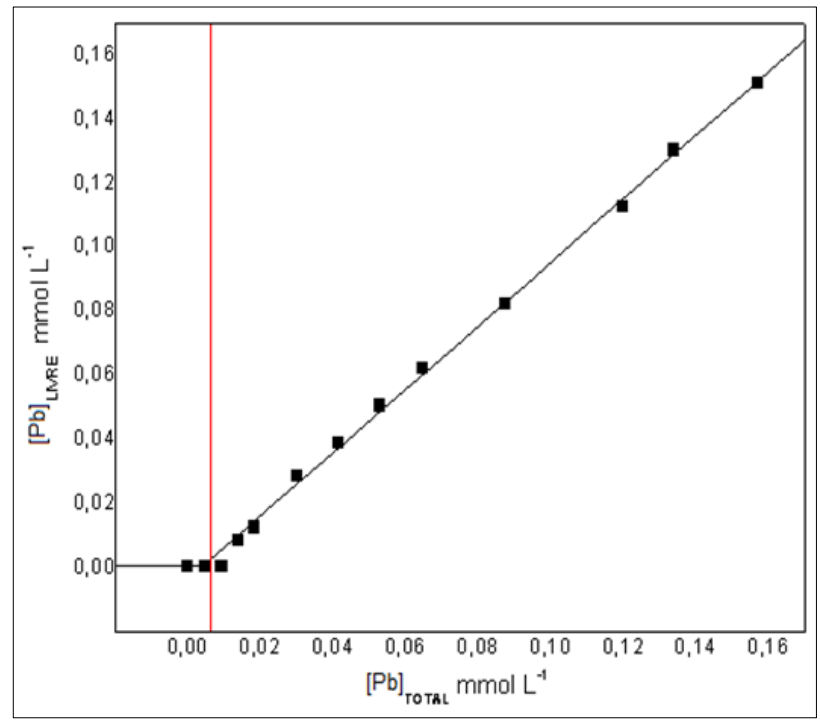

Fonte: Dados experimentais

Um estudo realizado por Abate e Masini (1999), utilizando SH do tipo comercial e isoladas dos sedimentos do reservatório de Barra Bonita, cerca de 270 km da cidade de São Paulo, estudou-se o emprego de eletrodos de amálgama de $\mathrm{Pb}$, Cd e Zn para avaliar a capacidade de complexação dos respectivos metais. Em tal estudo, concluíram que foi uma técnica eficiente em relação à sensibilidade e reprodutibilidade para estudos de propriedades de complexação de SH. Ainda de acordo com os pesquisadores, tanto a amostra do tipo comercial quanto a do reservatório apresentaram capacidade de complexação muito semelhantes, sendo o maior grau de complexação observado e comparado com o nosso estudo foi também $\mathrm{o} \mathrm{Pb}$.

\section{CONSIDERAÇÕES FINAIS}

Os riscos do descarte indiscriminado de resíduos contendo metais potencialmente tóxicos ao solo estão associados principalmente ao transporte e destinação desses poluentes no ambiente. Assim, a natureza estrutural das SH da turfa estudada favorece a complexação dos íons metálicos introduzidos antropicamente no ambiente.

Assim, os resultados desse trabalho mostram que a matéria orgânica está exercendo uma importante função, complexando os íons metálicos e agindo como uma barreira de proteção natural, minimizando temporariamente o impacto ambiental causado pela lixiviação de metais.

\section{REFERÊNCIAS}

ABATE, G.; MASINI, J. C. The use of potentiometric amalgam electrodes for complexation studies of humic substances. Química Nova, v. 22, n. 5, p. 661-665, 1999. 
https://doi.org/10.1590/S0100-

$\underline{40421999000500006}$

BEZERRA, P. S. S.; TAKIYAMA, L. R.; BEZERRA, C. W. B. Complexação de íons de metais por matéria orgânica dissolvida: modelagem e aplicação em sistemas reais. Acta Amazonica, Manaus, v. 39, n. 3, p. 639-648, 2009. https://doi.org/10.1590/S0044-

$\underline{59672009000300019}$

BOTERO, W. G.; SANTOS, A.; ROCHA, J. C. Caracterização de lodo gerado em estações de tratamento de água: perspectivas de aplicação agrícola. 2008. 68 f. Dissertação (Mestrado) - Universidade Estadual Paulista, Instituto de Química, 2008. Disponível em: <http://hdl.handle.net/11449/97748>.

CADORE, S.; MATOSO, E.; SANTOS, M. C. A espectrometria atômica e a determinação de elementos metálicos em material polimérico. Quím. Nova, São Paulo, v. 31, n. 6, p. 15331542,2008. https://doi.org/10.1590/S010040422008000600044.

CETESB. FIT- Ficha de Informação Toxicológica. Cádmio. 2012a. Disponível em: <http://laboratorios.cetesb.sp.gov.br/wpcontent/uploads/sites/47/2013/11/cadmio1.pdf >. Acesso em: 22 nov. 2016.

CETESB. FIT- Ficha de Informação Toxicológica. Chumbo. 2012b. Disponível em: <http://laboratorios.cetesb.sp.gov.br/wpcontent/uploads/sites/47/2013/11/chumbo. pdf>. Acesso em: 22 nov.2016.

DERISIO, J. C. Introdução ao controle de poluição ambiental. 4. ed. Oficina de Textos, 2016.

GOMES, V. M.; SANTOS, A.; OLIVEIRA, L. C. Estudo da mobilidade do crômio em área de descarte inadequado de resíduos de couro: possível contaminação de solos, águas e sedimentos. 2010. 83 f. Dissertação (Mestrado) - Universidade Estadual Paulista,
Instituto de Química, 2010. Disponível em: <http://hdl.handle.net/11449/97798>.

MENDONÇA, A. G. R. Turfa: interações com espécies metálicas e estudo de parâmetros que influenciam na utilização em setores agrícolas. 2010. 158 f. Tese (Doutorado em Química Analítica) - Instituto de Química, Universidade Estadual Paulista, Araraquara, 2010.

MOREIRA, F. R.; MOREIRA, J. C. A importância da análise de especiação do chumbo em plasma para a avaliação dos riscos à saúde. Química Nova, São Paulo , v. 27, n. 2, p. 251-260, Abr. 2004. Acesso em: 09 Abr. 2017. https://doi.org/10.1590/S010 0-40422004000200015.

NATUME, R. Y. et al. Gerenciamento de resíduos de informática nas universidades federais do Brasil. In: COLÓQUIO INTERNACIONAL SOBRE GESTÃO UNIVERSITÁRIA NA AMÉRICA DO SUL E II CONGRESSO INTERNACIONAL IGLU, 11. Anais... 2011.

OLIVEIRA, B. O. S. et al. Teores de metais pesados e parâmetros do solo e da água em área de disposição de resíduos sólidos urbanos em Humaitá-AM. Engenharia Sanitária e Ambiental, v. 21, n. 3, 2016. https://doi.org/10.1590/S1413$\underline{41522016133274}$

PEARSON, R. G. Hard and soft acids and bases.J. Am. Chem. Soc., v. 85,p. 3533-3539, 1963. https://doi.org/10.1021/ja00905a001

ROCHA, J. C.; ROSA, A. H. Substâncias húmicas aquáticas: interações com espécies metálicas. São Paulo: UNESP, 2003. 138 p.

ROCHA, J. C.; ROSA, A. H.; CARDOSO, A. A. Introdução à química ambiental. 2. ed. Porto Alegre: Bookman, 2009.

ROMÃO, L. P. C. et al. Tangential-flow ultrafiltration: a versatile methodology for 
determination of complexation parameters in refractory organic matter from Brazilian water and soil samples. Anal. Bioanal. Chem., v. 375, n. 8, p. 1097-1100, 2003. https://doi.org/10.1007/s00216-002-1728-6

ROSA, A. H. et al. Oxidação das substâncias húmicas de turfa durante o processo de extração alcalina. Estudo espectroscópico na região do infravermelho e do visível. In: CONGRESSO BRASILEIRO DE QUÍMICA, 27. Anais... Natal, 1998. v. 47, p. 25-28.

SANTOS, A. Interações entres espécies metálicas e substâncias húmicas extraídas de solo da micro bacia de drenagem no reservatório de captação de águas Anhumas - Araraquara-SP. 2003. 110 f. Tese (Doutorado em Química) - Instituto de Química de São Carlos, Universidade de São Paulo, São Carlos, 2003. 